\title{
Investigation of the microstructure of milk protein concentrate powders during rehydration: Alterations during storage
}

\author{
A. Mimouni, ${ }^{*}$ H. C. Deeth, ${ }^{*}$ A. K. Whittaker,† M. J. Gidley,‡ and B. R. Bhandari* \\ *School of Land, Crop and Food Sciences, The University of Queensland, Brisbane 4072, Australia \\ †Centre for Magnetic Resonance, and Australian Institute for Bioengineering and Nanotechnology (AIBN), The University of Queensland, \\ Brisbane 4072, Australia \\ ¥Centre for Nutrition and Food Sciences (CNAFS), The University of Queensland, Brisbane 4072, Australia
}

\section{ABSTRACT}

The aim of this work was to use scanning electron microscopy to investigate the microstructure of rehydrated milk protein concentrate powder (MPC) particles. A sample preparation method for scanning electron microscopy analysis of rehydrated MPC particles is described and used to characterize the time course of dissolution and the effects of prior storage on the dissolution process. The results show that a combination of different types of interactions (e.g., bridges, direct contact) between casein micelles results in a porous, gel-like structure that restrains the dispersion of individual micelles into the surrounding liquid phase without preventing water penetration and solubilization of nonmicellar components. During storage of the powder, increased interactions occur between and within micelles, leading to compaction of micelles and the formation of a monolayer skin of casein micelles packed close together, the combination of which are proposed to be responsible for the slow dissolution of stored MPC powders.

Key words: scanning electron microscopy, microstructure, powder, casein micelle

\section{INTRODUCTION}

Milk protein concentrate (MPC) is a high-protein milk powder containing up to $85 \%$ protein. These powders are produced from skim milk by spray-drying after pasteurization, ultrafiltration, diafiltration, and vacuum evaporation (Havea, 2006; Singh, 2006). They are typically used by the food industry as gelling, foaming, or emulsifying ingredients as well as for their nutritional value. For most applications, prior dissolution of MPC in water is needed, preferably at room temperature and ideally as fast as possible with moderate agitation to minimize operating costs. Because the powder does not fully express its functional properties if it remains in-

Received May 11, 2009.

Accepted August 6, 2009.

${ }^{1}$ Corresponding author: b.bhandari@uq.edu.au soluble, a true solution or complete dispersion of colloidal particles is required (Gaiani et al., 2007; Martin et al., 2007). However, commercial MPC powders, freshly made or stored, are characterized by poor reconstitution properties (Schuck et al., 1994; Singh, 2006; Baldwin and Truong, 2007; Mimouni et al., 2009). The step of dissolution of powder particles, rather than wetting or deagglomeration, has been shown to be the ratelimiting step in rehydration (Mimouni et al., 2009) with storage-induced effects further altering the dissolution kinetics (A. Mimouni, unpublished data). Some form of association of micelles upon storage may be responsible for these alterations (McKenna, 2000; Anema et al., 2006; Havea, 2006). However, further work is needed to understand the structural parameters controlling the dissolution rate of MPC powder particles.

Scanning electron microscopy has been used to study various types of dairy powders by several researchers (Thomas et al., 2004; Gaiani et al., 2007; Tamime et al., 2007b). However, to the best of our knowledge, these investigations were only performed on spray-dried powders in the dry state and did not provide information directly on rehydrated structures. The change in the structure of powder particles during the course of dissolution can provide valuable information on the dispersion mechanism of the colloidal protein particles. Therefore, the aim of this work was to use scanning electron microscopy as a tool to investigate the microstructure of rehydrated MPC powder particles. We present a sample preparation method for electron microscopy analysis that was adapted from different existing protocols. The observed microstructure of MPC powder and its alterations upon storage are described and then discussed in relation to the rehydration properties of the powder.

\section{MATERIALS AND METHODS}

\section{Milk Protein Concentrate Powder}

The chemical composition of MPC used in this study (MPC85, Murray Goulburn Co-operative Co. Limited, 
Brunswick, Victoria, Australia) was analyzed by the manufacturer using the methods described in Standards Australia (1995). This MPC primarily contains milk proteins $(82.4 \% \mathrm{wt} / \mathrm{wt})$ with the same proportion of casein and whey proteins as in the milk used for the powder manufacture, lactose (4\% wt/wt), ash $(7.3 \% \mathrm{wt} / \mathrm{wt})$, and fat $(1.6 \% \mathrm{wt} / \mathrm{wt})$. Immediately after manufacture, the powder was transported to the laboratory where it was stored at $4^{\circ} \mathrm{C}$ until used. Before rehydration, the powder was removed from the cold room and stored for $2 \mathrm{~d}$ at $20^{\circ} \mathrm{C}$ in plastic desiccators (250-mm diameter) above a saturated potassium acetate solution (equilibrium relative humidity $23 \%$ ) to ensure moisture content standardization. Changes in powder microstructure occurring during storage were also investigated. For this purpose, MPC85 powder was stored for $2 \mathrm{mo}$ at the same temperature $\left(20^{\circ} \mathrm{C}\right)$ and relative humidity $(23 \%)$.

\section{Rehydration Procedure}

Rehydration was carried out at 5\% (wt/wt) powder concentration in water. The MPC85 powder (10 g) was added to $190 \mathrm{~g}$ of water in a $250-\mathrm{mL}$ glass beaker. The suspension was maintained at constant temperature using a water bath at $24^{\circ} \mathrm{C}$. Stirring was performed for 10 min (short-term rehydration) and $80 \mathrm{~min}$ (long-term rehydration) at constant speed (200 rpm) using an electric overhead mixer (RW20, IKA, Staufen, Germany) and a 4-bladed propeller stirrer of 50-mm diameter (R 1342, IKA; Mimouni et al., 2009).

\section{Sample Preparation for Scanning Electron Microscopy and Imaging}

The MPC powder was rehydrated following the procedure presented above. To capture powder particles in suspension, particles were adhered to a silicon chip wafer (ProSciTech, Kirwan, Queensland, Australia) coated with poly-L-Lys (Sigma, Castle Hill, New South Wales, Australia), which creates electrostatic bonding between the powder particles and the substrate (McMahon and Oommen, 2008). A few drops of poly-L-Lys solution were deposited on the silicon wafer and allowed to airdry at room temperature (i.e., approximately $20^{\circ} \mathrm{C}$ ) in a dust-free environment. One or two drops of MPC suspension were then deposited and left for 5 min before draining and rinsing the wafer with $100 \mathrm{~m} M$ phosphate buffer ( $\mathrm{pH} 7)$.

A solution of $3 \%$ glutaraldehyde in $100 \mathrm{~m} M$ phosphate buffer ( $\mathrm{pH} 7$ ) was then applied for 15 min to achieve chemical fixation of the rehydrated protein material. After fixation, the samples were washed in deionized water (3 times) and dehydrated using a graded ethanol series: 50, 70, 90, 95 (2 times), and 100\% (3 times). The elapsed time per solution was 5 min (Dalgleish et al., 2004). Samples were then dried using $\mathrm{CO}_{2}$ in a critical point dryer (CPD 010, Balzers Union Ltd., Liechtenstein). The silicon wafer was subsequently mounted onto electron microscopy stubs by placing it on a carbon double-sided adhesive tape. Protein powders suffer from extensive charge buildup under the electron beam, and hence they need to be coated with conductive material. Samples were platinum-coated for $2 \mathrm{~min}$ (to about $5 \mathrm{~nm}$ thick) and examined with a field emission scanning electron microscope (6300, Jeol, Tokyo, Japan) operating at 2.5, 5, 10, or $15 \mathrm{kV}$. For analysis of the spray-dried powder, the powder samples were placed on the carbon double-sided tape mounted onto electron microscopy stubs. The images were produced in the Centre for Microscopy and Microanalysis of the University of Queensland (St Lucia, Queensland, Australia).

\section{RESULTS AND DISCUSSION}

\section{Microstructure of Fresh Powder Particles}

Effects of Short-Term Rehydration. Figure 1 shows the difference in surface microstructure between spray-dried (A) and rehydrated (B) milk protein powder particles rehydrated for 10 min. Before rehydration (Figure 1A), the surface of the particle was essentially smooth, without any visible substructures on the surface. An increase in magnification did not reveal additional structural details. Note that the scanning electron microscope can achieve a very high resolution with spray-dried materials. Some holes and roughness on the surface of the particles measuring less than 500 $\mathrm{nm}$ were visible, which suggests that the smooth surface observed was not the consequence of a lack of resolution but seems to represent the true surface of spraydried powder particles. A smooth surface appearance was demonstrated in previous work (Tamime et al., 2007b) and was attributed to the strong compaction and shrinkage of the protein material, especially the casein micelles, during air-drying. In contrast, the hydration of powder particles (i.e., the presence of water) considerably modified the 3-dimensional organization of the material on the surface of the powder particles. The microstructure of a powder particle (Figure 1, panels B, C, and D) was porous and characterized by a loose assembly of spherical nanoparticles grouped in small clusters separated by pores of various sizes. The diameter of these spheres was in the range of 40 to 200 $\mathrm{nm}$, which strongly suggests that these particles are casein micelles. The pore diameter as well as the size of the casein micelle clusters were both in the approxi- 
mate range of 100 to $300 \mathrm{~nm}$ and sometimes smaller. The surface microstructure of MPC powder particles showed similarities with that reported for gels obtained from native phosphocaseinate suspension after acidification at $\mathrm{pH} 4.6$ with glucono- $\delta$-lactone (Le Feunteun and Mariette, 2007). However, the observed surface microstructure exhibited some important differences with the microstructure of glucono- $\delta$-lactone acid skim milk gels reported by Sanchez et al. (2000) or with the structure of a set yogurt (Tamime et al., 2007a). The images reported in those articles revealed a well-structured and open network (Sanchez et al., 2000; Tamime et al., 2007a) composed of casein micelles grouped in small clusters and strands separated by large pores of homogeneous size. In contrast, the microstructure of hydrated MPC powder particles reported in the present study (Figure 1, panels B, C, and D) can be regarded as a coarse network or as an organization intermediate between a random aggregated particulate structure and a full gel network.

When viewed at high magnification (Figure 1, panels $\mathrm{C}$ and $\mathrm{D}$ ), the casein micelles appeared to be linked either by direct intermicellar contacts or by short bridges composed of material protruding from the surface of micelles. The physicochemical nature of these links is unknown and will need further investigation.

Effects of Long-Term Rehydration. The scanning electron micrographs in Figure 2 show the structure of the powder particles rehydrated for $80 \mathrm{~min}$ at $24^{\circ} \mathrm{C}$. It is first worth noting that powder particles in the approximate range of 10 to $40 \mu \mathrm{m}$ were present. This size range was similar to that observed for particles rehydrated
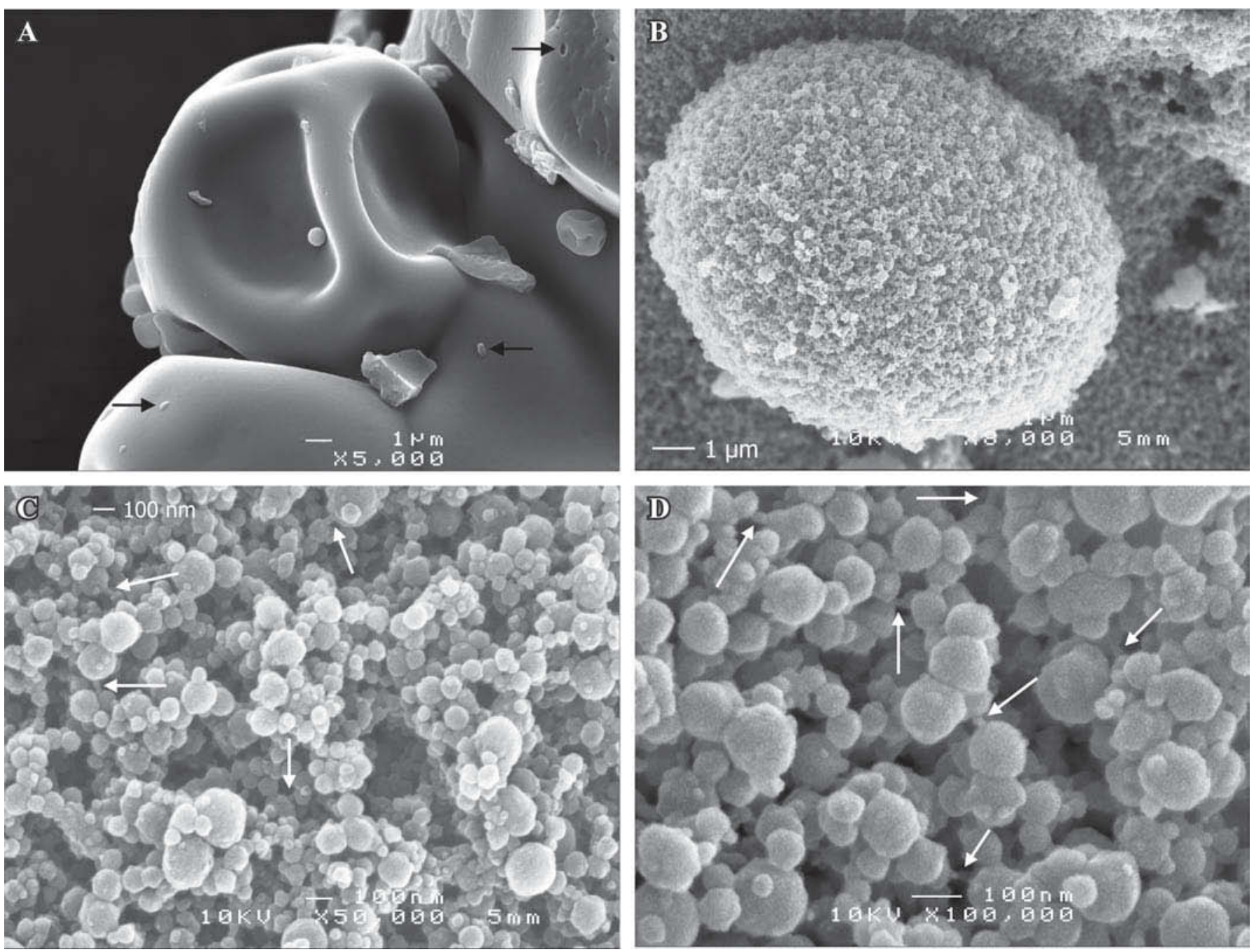

Figure 1. Field emission scanning electron micrographs of fresh milk protein concentrate powder particles after rehydration for 10 min: A) initial spray-dried powder particle; B) rehydrated powder particle; C, D) details of the surface of rehydrated powder particles at 2 magnifications $(\times 50,000$ and $\times 100,000)$. The black arrows in panel A indicate the presence of $\sim 100$-nm holes and surface roughness. The white arrows in panels $\mathrm{C}$ and $\mathrm{D}$ indicate the presence of intermicellar bridges. 

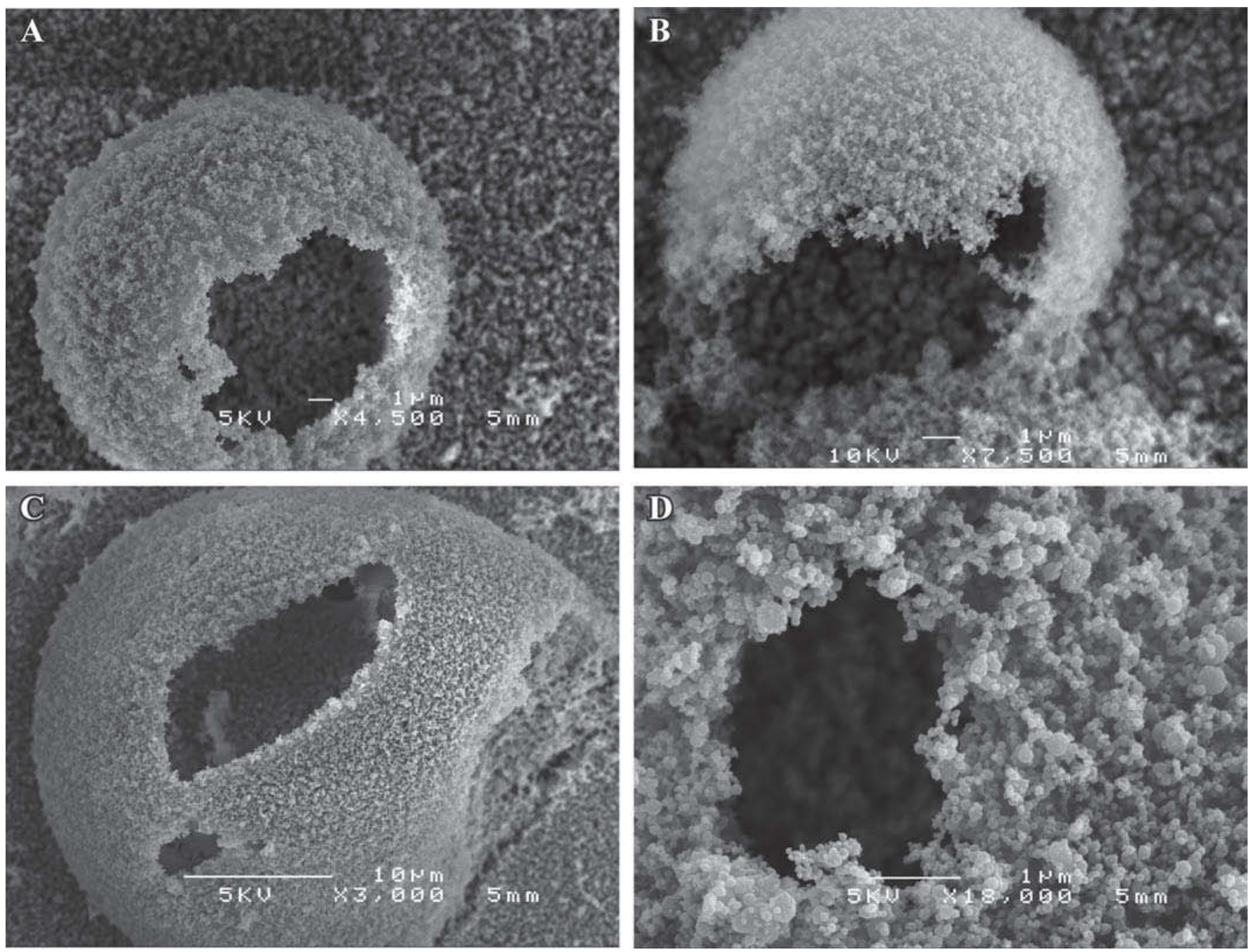

Figure 2. Field emission scanning electron micrographs of fresh milk protein concentrate powder particles after rehydration for 80 min observed at different magnifications: A) $\times 4,500$; B) $\times 7,500$; C) $\times 3,000$; and D) $\times 18,000$.

for $10 \mathrm{~min}$ (Figure 1B). For the remaining undissolved powder particles, large defects within the surface were also observed after long-term rehydration, providing an insight into the hollow interior of the particles. At an advanced stage of rehydration, the particles were composed of only a thin external layer of material, which was breached at certain points (Figure 2B). Judging from the SEM micrographs of Figure 2, this external layer has the appearance of a fragile framework that precariously supports the spherical structure of the particles on the verge of collapsing and fully dispersing. At higher magnification, the characteristics of the casein micelle network-like organization on the surface of the particles rehydrated for 80 min (Figure 2D) were very similar to those observed after 10 min of rehydration (Figure 1). This result suggests that dissolution of the powder particles occurred by gradual erosion from both external surfaces and internal surfaces exposed by breaches in the outer layer.

\section{Microstructure of Aged Powder Particles}

Effects of Short-Term Rehydration. The MPC85 powder was stored for 2 mo (aged) at $24^{\circ} \mathrm{C}$ and $23 \%$ equilibrium relative humidity. Comparing the microstructure of spray-dried powder particles before and after storage reported in Figures 1A and 3A, respectively, no visible changes were observed. Both aged and fresh MPC85 powder particles exhibited a smooth surface without any detectable substructures (e.g., casein micelles). However, very significant differences between fresh and aged powder particles appeared when the powder was rehydrated (Figure 3, panels B, C, and D). The most striking difference was the increase in the 

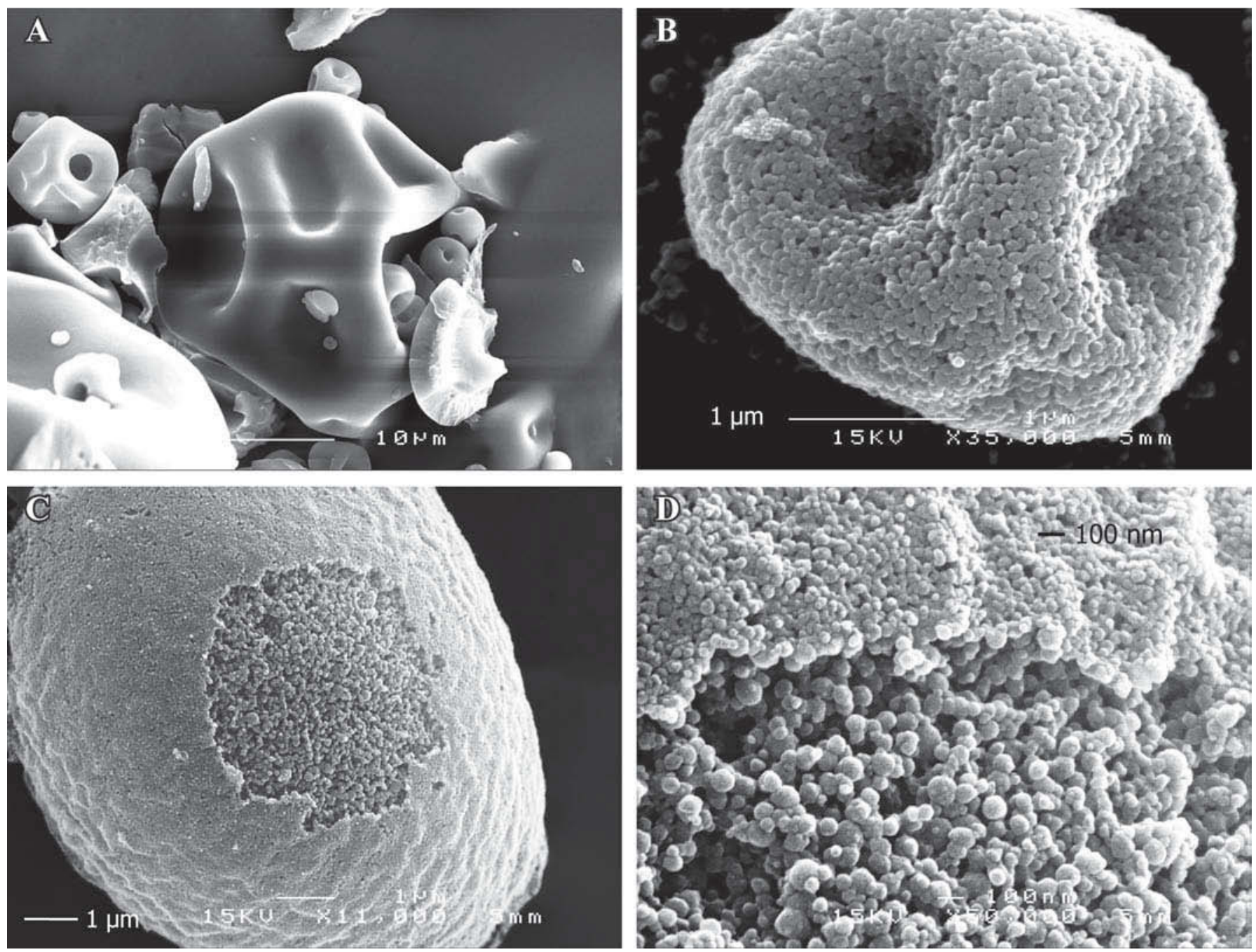

Figure 3. Field emission scanning electron micrographs of aged milk protein concentrate powder particles after 2 -mo storage at $20^{\circ} \mathrm{C}$ and water activity of 0.23 : A) spray-dried powder particle; B, C) hydrated powder particle after rehydration for 10 min; D) details of the surface of the rehydrated powder particle $(\times 50,000)$ observed in panel $\mathrm{C}$.

density and compaction of the casein micelles on the surface of the aged powder particles, which left almost no space between the sphere-like particles (Figure 3B). The surface of the aged powder particles (Figure 3C) did not have the characteristic open gel-like organization observed for the fresh powder (Figure 1C). Moreover, large breaches were often observed in the external layer of the powder particles indicating the existence of 2 levels of organization (Figure 3 panels $\mathrm{C}$ and $\mathrm{D}$ ): underneath a peripheral layer of tightly packed casein micelles forming a skin on the outside of the particle, a more open and porous spatial organization was apparent. This internal organization was similar to that of fresh MPC85 powder particles, although exhibiting lower porosity and a higher apparent extent of interactions between micelles. One can also notice that the micelles were slightly larger in fresh powder particles than in the inside of aged particles, which themselves were larger than in the skin-like external layer. These differences of size suggest differences in the extent of expansion during hydration of the micelles themselves.

Effects of Long-Term Rehydration. The micrographs in Figure 4 show the structure of the aged powder particles rehydrated for $80 \mathrm{~min}$ at $24^{\circ} \mathrm{C}$. In contrast to the early stage of rehydration in which most of the particles displayed an intact skin-like structure on their surface (Figure 1B), most of the particles observed after long-term rehydration exhibited partial (Figure 4, panels A and B) or almost total removal of the crust (Figure 4, panels $\mathrm{C}$ and D). On the basis of these images, this crust seemed to have become detached upon rehydration. Its disruption would not have occurred by gradual dissociation into individual micelles but by removal of large pieces (Figure 4B), suggesting strong 

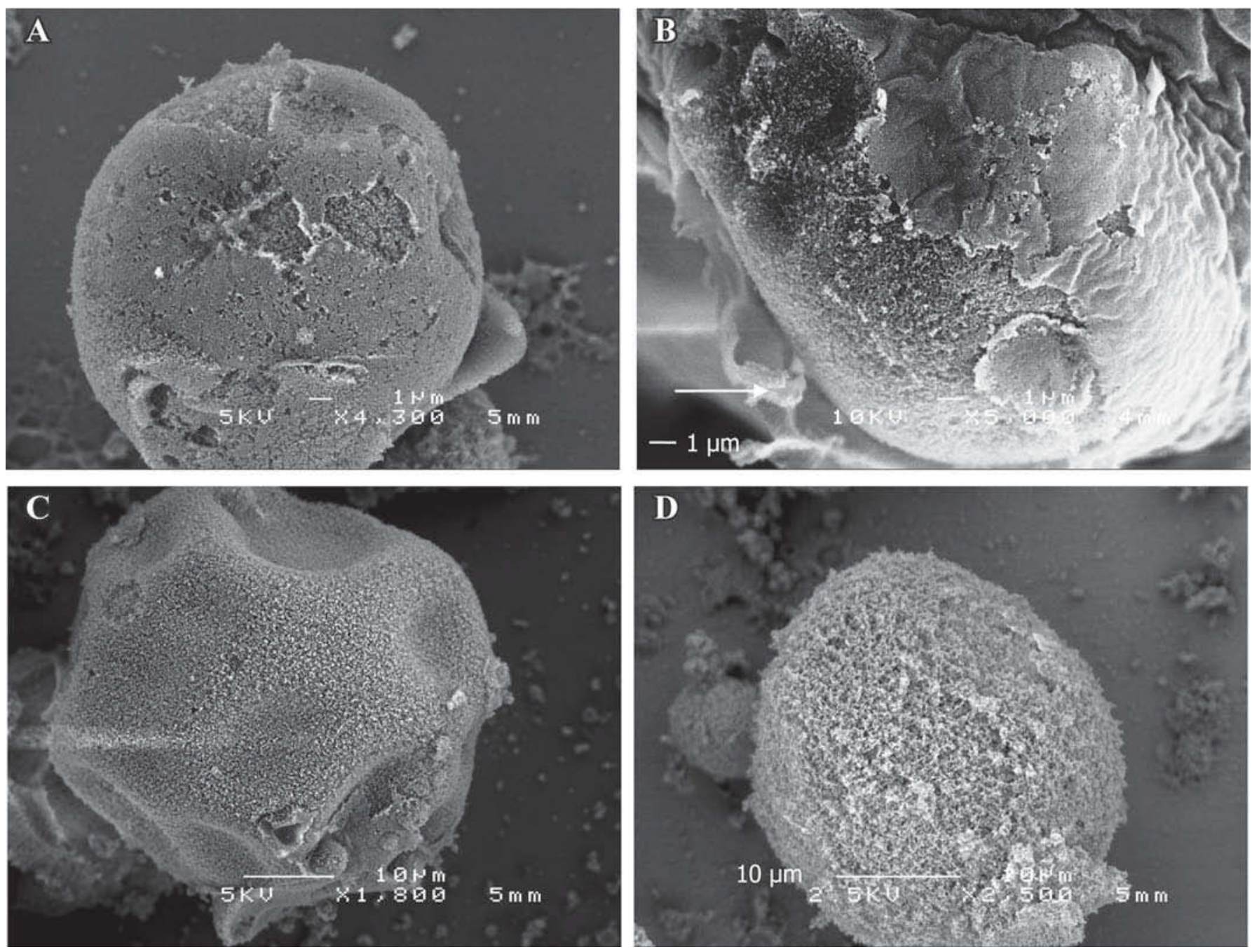

Figure 4. Field emission scanning electron micrographs of aged milk protein concentrate powder particles after rehydration for 80 min observed at different magnifications: A) $\times 4,300$; B) $\times 5,000$; C) $\times 1,800$; and D) $\times 2,500$. The arrow indicates part of the crust undergoing disruption.

micelle-micelle interactions within the crust, possibly irreversible upon rehydration.

Additionally, the substrate around the aged particles in Figure 4, panels $\mathrm{A}, \mathrm{C}$, and $\mathrm{D}$ was not covered by dispersed casein micelles, in contrast to the substrate around the fresh particles after the same period of rehydration (Figure 2). These observations strongly suggest that the dissolution of aged MPC powder particles was much slower and more inhibited than that of their fresh counterparts.

\section{Possible Artifacts of Sample Preparation}

Investigating microstructure by electron microscopy is not straightforward, especially in the case of hydrated specimens, and many artifacts may arise from the differ- ent steps involved in sample preparation (Hermansson and Buchheim, 1981; McMahon and McManus, 1998; Karlsson et al., 2007). First, some alteration of the native structure of the specimen can occur during surface immobilization by physical adsorption of the casein micelles onto the substrate (Dalgleish et al., 2004; Martin et al., 2006). The electrostatic interactions may then induce spreading and distortion of the micelles in direct contact with the poly-L-Lys-coated wafer. These interactions are, however, unlikely to affect the structure of the powder particle surface located several micrometers from the substrate (Figure 1B).

The removal of liquid water is required for imaging under the vacuum of the microscope (Spagnuolo et al., 2005). To withstand this drying process without changing the native structure of the particles, a prior chemical 
fixation step is necessary (Hermansson and Buchheim, 1981). We used glutaraldehyde, which is considered a preferred crosslinking agent to formaldehyde (McMahon and McManus, 1998). Osmium tetroxide was not used because it seems to have no additional beneficial effect in the preparation of casein micelle samples (Martin et al., 2006). McMahon and Oommen (2008) mentioned that fixation with glutaraldehyde probably affects the structure of casein micelles and may not be suitable for analyzing the internal organization. In contrast, several recent studies reported application of fixatives such as glutaraldehyde to analyze casein micelle-к-carrageenan interactions (Spagnuolo et al., 2005), micelle-micelle interactions, and subsequent network organization (Sanchez et al., 2000; Le Feunteun and Mariette, 2007) without mention of artifacts caused by chemical fixation. We conclude that glutaraldehyde fixation may affect the internal structure of the micelles but should not alter the extent and characteristics of the native interactions between micelles. In support of this, Karlsson et al. (2007) demonstrated, in ultrafiltered skim milk concentrate analyzed by transmission electron microscopy, that although casein micelles were close to each other, no signs of merged micelles or of pronounced aggregation were observed despite the use of glutaraldehyde to fix the specimen. In addition, when investigating $\kappa$-carrageenan-micelle interactions using scanning electron microscopy, Spagnuolo et al. (2005) ruled out the possibility that glutaraldehyde induced crosslinking or bridging between neighboring micelles, which was based on the lack of microscopic evidence and on dynamic light scattering data that showed no increase in casein micelle diameter caused by the fixatives used. Taken together, these results strongly suggest that the bridges and the gel-like organization observed in Figure 1, panels B, C, and D were not artifacts caused by glutaraldehyde. Moreover, the washing step between material immobilization and fixation would have removed the micelles held solely by surface tension (McMahon and Oommen, 2008). Consequently, even though chemical fixation may have linked the micelles in contact with each other as suggested by Gastaldi et al. (1996), these contacts must have resulted from preexisting (i.e., native) interactions that were strong enough to withstand the washing procedure.

The postfixation dehydration methods used in this study (i.e., graded ethanol followed by $\mathrm{CO}_{2}$ criticalpoint drying) have been used recently to analyze casein micelles and casein gel structure (Sanchez et al., 2000; Dalgleish et al., 2004; Spagnuolo et al., 2005; Martin et al., 2006, Le Feunteun and Mariette, 2007) and are standard procedures for biological materials (Hayat, 2000). These methods were reported to give the best possible images achievable using state-of-the-art tech- nology (Dalgleish et al., 2004) by causing minimal alteration of the structure from the native state. Finally, although metal coating is known to hide the fine details of the surface of casein micelles and to give to the latter a lumpy appearance (McMahon and McManus, 1998), there is no mention in the literature of any artifacts caused in the organization of casein micelle networks. Coating is indeed a common procedure (Sanchez et al., 2000; Spagnuolo et al., 2005; Martin et al., 2006, Le Feunteun and Mariette, 2007). Thus, the pore widths, cluster size, and extent and characteristics of micellemicelle interactions observed in Figures 1, 2, 3, and 4 should not have been significantly affected by metal coating.

The changes in the microstructure of MPC85 powder particles after storage are also unlikely to be artifacts of sample preparation. This is supported by the fact that the presence of a monolayer of tightly packed casein micelles forming a skin on the outside of the particle as seen in Figure 3 was visible on samples prepared with the same protocol and within the same batch of criticalpoint drying as fresh particles (Figure 1). Moreover, there are no simple arguments to explain why sample preparation would affect different parts of the surface of the same particle differently. Therefore, the presence of both types of structure (i.e., compacted and loose assembly of micelles) on the same particles (Figures 3 and 4) further confirms that these structures exist simultaneously in the native specimen and are not caused by sample preparation. Finally, particles with intact skinlike structure (Figure 3B) were visible among particles exhibiting partial detachment of this skin (Figure 2C). Further removal of the crust was observed on powder particles rehydrated for a longer period (Figure 4). For these reasons, it is highly probable that the detachment of this crust was induced by powder rehydration rather than sample preparation

\section{Relating the Microstructure and Rehydration Properties of MPC}

As observed in the micrographs of Figure 1 depicting the microstructure of fresh MPC85 powder particles after short-term rehydration, short bridges and direct intermicellar contacts act together to maintain a network that may stabilize the structure of the powder particles, making them difficult to disperse into individual micelles. Moreover, this structure is persistent after a longer period of rehydration (Figure 2), which further confirms its ability to resist disruption and its tendency to retard the release of protein material into the solvent. These results are consistent with the low dissolution rate of the particles shown to govern the rehydration rate of MPC85 (Mimouni et al., 2009). The 
results are also in accordance with the low solubility of MPC powders extensively demonstrated in the literature (Jimenez-Flores and Kosikowski, 1986; Schuck et al., 1994, 2007; Anema et al., 2006; Havea, 2006; Singh, 2006; Baldwin and Truong, 2007).

Remarkably, the microstructure of fresh hydrated MPC85 powder particles is relatively porous as shown in Figure 1, panels B, C, and D. The diameter of the pores was in the same range as the size of casein micelles, suggesting that steric hindrance may restrain the dispersion of micelles into the liquid phase. However, the pores are large enough to enable water to diffuse rapidly into the core of the particles and to allow nonmicellar solutes to diffuse out. This hypothesis was confirmed recently using a different experimental approach (A. Mimouni, unpublished data). In the latter study, nonmicellar solutes such as $\mathrm{Na}, \mathrm{K}$, and lactose as well as whey proteins were found to be released into solution rapidly and quantitatively during MPC85 powder rehydration. A similar conclusion was reached by Anema et al. (2006).

The characteristics of micelle-micelle attractive interactions observed in Figure 1, panels $\mathrm{C}$ and $\mathrm{D}$ are consistent with transmission electron micrographs of undissolved MPC powder particles in suspension reported by Havea (2006), which showed similar fibrillike structures linking micelles together. However, the physical and chemical nature of these linkages and the conditions of formation during MPC powder manufacture are not clearly established. These linkages could be composed of nonmicellar material ( $\kappa$-casein dissociated from the micelles, $\beta$-lactoglobulin) acting as a glue between the casein micelles (McKenna, 2000; Havea, 2006). The intermicellar bonds may involve weak forces (hydrophobic or hydrogen bonds) that would be reversible upon reconstitution, rather than covalent bonds such as disulfide interactions (Anema et al., 2006; Havea, 2006), as long-term rehydration results in almost complete solubilization (A. Mimouni, unpublished data). Another possibility that arises from our observations is that the linkages consist of micellar appendages protruding from the micelles themselves. The bridges observed in Figure 1 indeed are structurally similar to micellar tubule-like structures described by Dalgleish et al. (2004). These tubules extended from micelles as they adsorbed to the hydrophobic carbon substrate.

According to McKenna (2000) and Singh (2006), regardless of the molecular origin and the forces involved, these intermicellar attractions would be more likely to occur once casein micelles have been destabilized and had their structure changed during powder manufacturing process. Ultrafiltration and diafiltration may play an important role in changing the nature of the micelle because they modify both the proteins-to-minerals ratio and the minerals concentrations. The resulting changes in salt equilibria between micellar and soluble phases could lead to the loss of colloidal calcium phosphate, causing loosening and swelling of casein micelles (McKenna, 2000; Singh, 2006). Their aggregation would eventually occur when packed together during water removal (concentration and drying). However, the macrostructural changes and surface aggregation of such products during rehydration will need very careful examination to observe real differences. The presence of primary particles in the approximate size range of 10 to $40 \mu \mathrm{m}$ after a long period of rehydration (Figure 2) and the evolution of microstructure upon reconstitution (Figures 1 and 2) supports the mechanism of powder rehydration proposed previously by us (Mimouni et al., 2009), based on analysis of light scattering data. The powder material, located in an external layer around an internal vacuole, is likely to disperse by gradual erosion of the surface. This erosion might thin the external layer of material, which becomes transparent at some point (Figure 2B). The concomitant formation of large breaks and holes (Figure 2) might weaken the external framework of the powder particles, leading eventually to their collapse or full dissolution.

After a period of storage, the microstructure exhibited important differences only discernable in hydrated powder particles (Figure 3, panels $\mathrm{B}, \mathrm{C}$, and D). The increase in the extent of micelle-micelle interactions led to the development of a monolayer skin of highly packed or even fused micelles, whereas the internal structure of the particles showed a higher porosity. These observations confirm and extend those made by McKenna (2000) on aged MPC powder using transmission electron microscopy. An explanation of such a difference of structure between the periphery and the inside of the particle had been proposed by the same author. The micelles on the outside of the particles would be forced together by their adsorption at the particle-air interface, enhancing protein-protein interactions and leading to noncovalent crosslinking of the micelles.

It is likely that the development of the skin is involved in the decrease in dissolution rate observed during storage (A. Mimouni, unpublished data). However, other questions arise from these observations. Although the skin appears dense enough to inhibit the release of the micelles from the core of the particles (Figure 3B), it does not appear continuous enough to be impermeable to solutes or water, as stated in previous studies (Schuck et al., 1994; McKenna, 2000). In accordance with the observations in this paper, Anema et al. (2006) showed that penetration of water and release of solutes were not prevented even after storage of MPC85.

In addition, large breaks in the external skin often appeared on powder particles (Figure 3, panels $\mathrm{C}$ and 
D). Agitation and hydration of the particles during powder reconstitution are believed to weaken the external microstructure, leading to partial or almost complete disruption of the crust after some time, as shown in Figure 4. Consequently, the more porous internal part of the particle is brought into direct contact with water. However, no signs of further micelle dispersion were detected (e.g., absence of dispersed individual micelles on the silicon wafer, Figure 4). Moreover, in previous work, MPC85 particles were submitted to shear and were fractured, exposing surfaces not covered by the skin (McKenna, 2000). In accordance with our own observations, no further dispersion was then visible. In addition, the network of micelles appeared denser in the internal part of the aged particles than in fresh particles (Figures $1 \mathrm{C}$ and $3 \mathrm{C}$ ). Taken together, these results strongly suggest that, in addition to the crust that tends to disrupt during rehydration, internal micelle-micelle interactions that developed during storage contributed to the decrease in the dissolution rate.

Finally, the decrease in size of the casein micelles after a period of storage indicated that their ability to rehydrate had been affected. In addition to the propensity of the casein micelles to interact with each other, alterations of their own native internal structure may also have occurred. Further work will be needed to investigate these transformations.

\section{CONCLUSIONS}

A specific sample preparation method has been developed to enable the use of scanning electron microscopy to investigate the microstructure of high-protein dairy powder particles during rehydration. We have shown that the combination of different types of interactions (i.e., bridges, direct contact) between casein micelles resulted in a porous, gel-like structure that may restrain the dispersion of individual micelles into the surrounding liquid phase. However, the pores between the micelles are large enough to enable water to diffuse quickly into the core of particles and to allow nonmicellar solutes to diffuse out. Performing scanning electron microscopy on rehydrated powder was shown to be a powerful technique to evaluate the marked changes occurring during storage. During storage of the powder, increased interactions occurred between micelles, leading eventually to the formation of a monolayer skin of casein micelles packed closely together. The role of this skin in inhibiting the dissolution of MPC powder particles and the nature of intermicellar interactions will need further investigation.

\section{ACKNOWLEDGMENTS}

The authors are grateful to the staff of the Centre for Microscopy and Microanalysis of The University of Queensland for their technical and scientific contribution in this study. This research was supported by Dairy Innovation Australia Ltd. (DIAL) and the Australian Research Council-Linkage project no. LP0669191.

\section{REFERENCES}

Anema, S. G., D. N. Pinder, R. J. Hunter, and Y. Hemar. 2006. Effects of storage temperature on the solubility of milk protein concentrate (MPC85). Food Hydrocoll. 20:386-393.

Baldwin, A. J., and G. N. T. Truong. 2007. Development of insolubility in dehydration of dairy milk powders. Food Bioprod. Process. 85:202-208.

Dalgleish, D. G., P. A. Spagnuolo, and H. D. Goff. 2004. A possible structure of the casein micelle based on high-resolution fieldemission scanning electron microscopy. Int. Dairy J. 14:10251031.

Gaiani, C., P. Schuck, J. Scher, S. Desobry, and S. Banon. 2007. Dairy powder rehydration: Influence of protein state, incorporation mode, and agglomeration. J. Dairy Sci. 90:570-581.

Gastaldi, E., A. Lagaude, and B. Tarodo De La Fuente. 1996. Micellar transition state in casein between $\mathrm{pH} 5.5$ and 5.0. J. Food Sci. 61:59-64.

Havea, P. 2006. Protein interactions in milk protein concentrate powders. Int. Dairy J. 16:415-422.

Hayat, M. A. 2000. Principles and Techniques of Electron Microscopy for Biological Applications. 4th ed. Cambridge University Press, New York, NY.

Hermansson, A. M., and W. Buchheim. 1981. Characterization of protein gels by scanning and transmission electron-microscopy-A methodology study of soy protein gels. J. Colloid Interface Sci. 81:519-530.

Jimenez-Flores, R., and F. V. Kosikowski. 1986. Properties of ultrafiltered skim milk retentate powders. J. Dairy Sci. 69:329339 .

Karlsson, A., R. Ipsen, and Y. Ardo. 2007. Observations of casein micelles in skim milk concentrate by transmission electron microscopy. Food Sci. Technol. 40:1102-1107.

Le Feunteun, S., and F. Mariette. 2007. Impact of casein gel microstructure on self-diffusion coefficient of molecular probes measured by H-1 PFG-NMR. J. Agric. Food Chem. 55:1076410772 .

Martin, A. H., H. D. Goff, A. Smith, and D. G. Dalgleish. 2006. Immobilization of casein micelles for probing their structure and interactions with polysaccharides using scanning electron microscopy (SEM). Food Hydrocoll. 20:817-824.

Martin, G. J. O., R. P. W. Williams, and D. E. Dunstan. 2007. Comparison of casein micelles in raw and reconstituted skim milk. J. Dairy Sci. 90:4543-4551.

McKenna, A. B. 2000. Effect of processing and storage on the reconstitution properties of whole milk and ultrafiltered skim milk powders. PhD Thesis. Massey University, Palmerston North, New Zealand.

McMahon, D. J., and W. R. McManus. 1998. Rethinking casein micelle structure using electron microscopy. J. Dairy Sci. 81:2985-2993.

McMahon, D. J., and B. S. Oommen. 2008. Supramolecular structure of the casein micelle. J. Dairy Sci. 91:1709-1721.

Mimouni, A., H. C. Deeth, A. K. Whittaker, M. J. Gidley, and B. R. Bhandari. 2009. Rehydration process of milk protein concentrate powder monitored by static light scattering. Food Hydrocoll. 23:1958-1965. 
Sanchez, C., R. Zuniga-Lopez, C. Schmitt, S. Despond, and J. Hardy. 2000. Microstructure of acid-induced skim milk-locust bean gumxanthan gels. Int. Dairy J. 10:199-212.

Schuck, P., S. Mejean, A. Dolivet, C. Gaiani, S. Banon, J. Scher, and R. Jeantet. 2007. Water transfer during rehydration of micellar casein powders. Lait 87:425-432.

Schuck, P., M. Piot, S. Mejean, J. Fauquant, G. Brule, and J. L. Maubois. 1994. Dehydration of an ultra-clean milk and micellar casein enriched milks. Lait 74:47-63.

Singh, H. 2006. Interactions of milk proteins during the manufacture of milk powders. Pages 413-423 in 27th World Dairy Congress and World Dairy Summit, IDF. EDP Sciences S. A., Shanghai, China.

Spagnuolo, P. A., D. G. Dalgleish, H. D. Goff, and E. R. Morris 2005. Kappa-caffageenan interactions in systems containing casein micelles and polysaccharide stabilizers. Food Hydrocoll. 19:371377.
Standards Australia. 1995. Methods of chemical and physical testing for the dairying industry-Introduction and lists of methods. AS 2300.0-1995. Stand. Aust., Sydney, Australia.

Tamime, A. Y.., A. Hassan, E. Farnworth, and T. Toba. 2007a. Structure of fermented milks. Pages 134-169 in Structure of Dairy Products. A. Y. Tamime, ed. Blackwell Publishing Ltd., Oxford, UK.

Tamime, A. Y., R. K. Robinson, and M. Michel. 2007b. Microstructure of concentrated and dried milk products. Pages 104-133 in Structure of Dairy Products. A. Y. Tamime, ed. Blackwell Publishing Ltd., Oxford, UK.

Thomas, M. E. C.. J. Scher, S. Desobry-Banon, and S. Desobry. 2004 Milk powders ageing: Effect on physical and functional properties. Crit. Rev. Food Sci. Nutr. 44:297-322. 